\title{
Outcomes of lung transplantation for cystic fibrosis in a large UK cohort
}

\author{
G Meachery, ${ }^{1}$ A De Soyza, ${ }^{2}$ A Nicholson, ${ }^{3}$ G Parry, ${ }^{1}$ A Hasan, ${ }^{1} \mathrm{~K}$ Tocewicz, ${ }^{1}$ T Pillay, ${ }^{1}$ \\ S Clark, J L Lordan, ${ }^{1,2}$ S Schueler, ${ }^{1}$ A J Fisher, ${ }^{1,2}$ J H Dark, ${ }^{1}$ F K Gould, ${ }^{3}$ P A Corris ${ }^{1,2}$
}

See Editorial, page 668, and page 732

${ }^{1}$ Department of

Cardiopulmonary

Transplantation, The Freeman

Hospital, High Heaton,

Newcastle-upon-Tyne, UK;

${ }^{2}$ Applied Immunobiology and

Transplantation, Institute of

Cellular Medicine, University of

Newcastle, UK; ${ }^{3}$ Department of

Medical Microbiology, The

Freeman Hospital, High Heaton,

Newcastle-upon-Tyne, UK

Correspondence to:

Dr A De Soyza, Applied

Immunobiology and

Transplantation, Institute of

Cellular Medicine, University of

Newcastle, Newcastle-upon-

Tyne NE7 7DN, UK;

anthony.de-soyza@ncl.ac.uk

GM and ADS contributed equally to the manuscript

Received 31 October 2007 Accepted 26 March 2008

Published Online First

16 May 2008

\section{ABSTRACT}

Background: Lung transplantation is an important option to treat patients with advanced cystic fibrosis (CF) lung disease. The outcomes of a large UK cohort of CF lung transplantation recipients is reported.

Methods: Retrospective review of case notes and transplantation databases.

Results: 176 patients with CF underwent lung transplantation at our centre. The majority (168) had bilateral sequential lung transplantation. Median age at transplantation was 26 years. Diabetes was common pretransplantation (40\%). Polymicrobial infection was common in individual recipients. A diverse range of pathogens were encountered, including the Burkholderia cepacia complex (BCC). The bronchial anastomotic complication rate was $2 \%$. Pulmonary function (forced expiratory volume in $1 \mathrm{~s} \%$ predicted) improved from a pretransplantation median of 0.8 I (21\% predicted) to 2.95 I (78\% predicted) at 1 year following transplantation. We noted an acute rejection rate of $41 \%$ within the first month. Our survival values were $82 \%$ survival at 1 year, $70 \%$ at 3 years, $62 \%$ at 5 years and $51 \%$ at 10 years. Patients with BCC infection had poorer outcomes and represented the majority of those who had a septic death. Data are presented on those free from these infections. Bronchiolitis obliterans syndrome (BOS) and sepsis were common causes of death. Freedom from BOS was $74 \%$ at 5 years and 38\% at 10 years. Biochemical evidence of renal dysfunction was common although renal replacement was infrequently required $(<5 \%)$.

Conclusion: Lung transplantation is an important therapeutic option in patients with CF even in those with more complex microbiology. Good functional outcomes are noted although transplantation associated morbidities accrue with time.

To date, lung transplantation is the most intensive therapeutic intervention used in the modern management of cystic fibrosis (CF). It remains the only therapeutic option that can restore patients with advanced cystic fibrosis lung disease towards normal respiratory health. Bilateral (sequential) cadaveric donor transplantation is currently the procedure of choice based on international data. ${ }^{1}$

Long term morbidity following lung transplantation is well described, both relating to obliterative bronchiolitis or bronchiolitis obliterans syndrome (BOS), ${ }^{1}$ immunosuppressant toxicity (eg, renal dysfunction $)^{2}$ and secondary malignancy. ${ }^{1}$ A number of single centre case series of lung transplantation for CF have been reported and demonstrate general excellent post-transplant survival results. ${ }^{3-6}$
As lung transplantation does not alter extrapulmonary manifestations of $\mathrm{CF}$, the burden of comorbidities related to the underlying CF are important considerations in long term survivors.

We were interested in assessing not only simple survival outcomes at our centre but also in defining functional outcomes, obliterative bronchiolitis rates and other major transplant associated comorbidities, such as renal dysfunction and malignancy rates. Hence our aim was to assess outcomes, including survival, and also the prevalence of transplantation associated comorbidities.

\section{METHODS}

A retrospective analysis of case notes, clinical charts and transplantation databases from the Freeman Hospital Cardiopulmonary Transplantation Unit was performed.

\section{Case finding and definitions}

We performed a retrospective analysis of case notes and the pulmonary transplantation database at our institution from 1989 (programme start) to the present date. We assessed all recipients with CF, including adult and paediatric cases, undergoing pulmonary transplantation, and reviewed their case notes and microbiological results. We have a comprehensive database of recipients with CF transplanted at our institution and cross referenced this with laboratory records to ensure the complete cohort was considered.

\section{Peritransplantation management}

We used a 3 day induction protocol with antithymocyte globulin (titrated by flow cytometric analysis of peripheral blood $\mathrm{T}$ lymphocytes) and intravenous methylprednisolone $(2 \mathrm{mg} / \mathrm{kg})$. All patients had triple immunosuppression post-transplantation comprising ciclosporin, prednisolone and azathioprine. Ciclosporin was commenced immediately post-transplantation if renal function was satisfactory. Alternative agents were used in the context of an international clinical trial (mycophenolate) or where intolerance of ciclosporin was noted (tacrolimus). Up to 5 days of intravenous ciclosporin was given in view of poor absorption of ciclosporin in CF. Prophylactic antibiotics were given to the recipient tailored to the most recent sensitivities derived from sputum cultures. If the isolate was panresistant, or recent information was unavailable, $2 \mathrm{~g}$ of aztreonam 8 hourly for 2-7 days was used. More recently, multiple antibiotic synergy testing has been incorporated into our microbiological workup of 
such patients using a previously described method. ${ }^{78}$ Aminoglycoside antibiotics were avoided in view of the potential synergistic toxicity with ciclosporin and loop diuretics. Patients were given an antistaphylococcal agent, flucloxacillin or clindamycin, in addition to metronidazole. We used prophylactic nebulised amphotericin or oral itraconazole/ voriconazole in patients who had evidence of pretransplantation Aspergillus infection with positive sputum cultures for this pathogen.

\section{Operative interventions}

These were performed as follows: heart-lung transplantation was performed via sternotomy with tracheal anastomosis and bicaval anastomosis. Bilateral (sequential) lung transplantation from a cadaveric donor was performed via clamshell incision with hilar anastomoses. In all cases the bronchial anastomosis was done with a mixture of continuous and interrupted monofilament nylon. Care was taken to place the anastomosis as close as possible to the lung parenchyma, no further than one cartilaginous ring from the bifurcation of the main bronchus. All bilateral lung transplantations were done with cardiopulmonary bypass. In some cases, bypass was used only during implant of the second lung, but both lungs were reperfused simultaneously. No procedures in this series were done without bypass. Aprotinin was used routinely. Living related lobar donor transplantation was also performed via clamshell incisions.

\section{Surveillance associated complications}

We routinely perform surveillance transbronchial biopsies and bronchoalveolar lavage at 1 week, 1 month, 3 months, 6 months and 1 year post-transplantation and at any time of unexplained deterioration. Major complications of transbronchial biopsy were recorded as present if there was requirement for chest drain insertion, biopsy associated bleeding with requirement for invasive ventilation or death following a procedure.

\section{Airway complications}

All our recipients underwent bronchoscopic and respiratory physiology assessment and monitoring for anastomotic complications. Airway complications such as stenosis, dehiscence and need for stenting were recorded from the case notes. Significant stenosis was deemed present if dilatation, stenting or debridement were required. ${ }^{6}$

\section{Obliterative bronchiolitis}

Pulmonary function tests were performed according to accepted guidelines. Forced expiratory volume in $1 \mathrm{~s}\left(\mathrm{FEV}_{1}\right)$ was performed on a Sensor Medics Autobox 6200 (Sensor Medics Corp, California, USA). The best consecutive $\mathrm{FEV}_{1}$ attained as directed by the guidelines was used to set thresholds for BOS 1 $\left(\mathrm{FEV}_{1} 66-80 \%\right.$ of best recorded post-transplantation $\left.\mathrm{FEV}_{1}\right), \mathrm{BOS}$ $2\left(\mathrm{FEV}_{1} 51-65 \%\right)$ and $\mathrm{BOS} 3\left(\mathrm{FEV}_{1}<50 \%\right)$. The more recently introduced category BOS 0 -p was not recorded (see discussion). We used "freedom from BOS" to define patients who failed to demonstrate a fall in $\mathrm{FEV}_{1}$ to the threshold used for BOS 1 or higher. ${ }^{9}$ If a patient underwent retransplantation, BOS rates were calculated until retransplantation, then further BOS analysis was based on standard criteria from the date of retransplantation.

\section{Survival analysis and causes of death recording}

Survival data are routinely collected as part of the national transplant surveillance programme. Actuarial survival analysis was conducted using StatView software V.4.5. The causes of deaths following transplantation were recorded from the notes. If there were doubts to the cause of death in the absence of a post mortem examination, two or more investigators reviewed the notes and a cause of death attributed based on supporting evidence in the notes. Sepsis related deaths were recorded where a pathogen was identified clinically and microbiologically as causal to the recipient's terminal decline or where a clinical diagnosis of infection was made and other possible diagnoses were excluded.

\section{Renal disease/dialysis requirements}

Although radioisotope measurement of renal function using ${ }^{51}$ chromium EDTA-glomerular filtration rate (GFR) forms part of the pretransplantation assessment, we do not use this technique to follow-up patients post-transplantation. Renal function was determined by serial serum creatinine levels recorded pretransplantation, at each routine clinic visit, including 1 year after transplantation, at 5 years and at 10 years. The number of patients receiving renal replacement therapy or who had undergone secondary renal transplantation was recorded from the case notes.

\section{Microbiology}

Peritransplantation microbiology from sputum and BAL of the recipient lung on the day of transplantation was recorded from the case notes/microbiology database. The presence or absence of bacterial coinfection based on microbial culture and the commercial phenotyping system API-20NE (Biomerieux, L'Etoile, France) species designation was recorded. Pulsed field gel electrophoresis assessment of microbiological clonality was not routinely conducted. Multiresistance and panresistance characteristics of Pseudomonas aeruginosa were recorded using previous definitions. ${ }^{10}$

\section{Malignancy}

Solid organ or haematological malignancy rates were identified from patient case notes and from cross referencing with

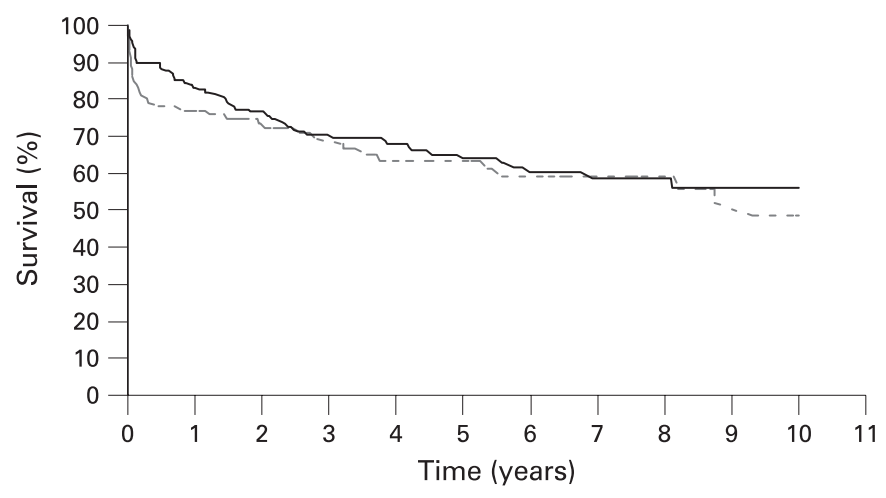

Figure 1 Actuarial survival of patients with cystic fibrosis (CF) at the Freeman Hospital Lung Transplant Programme compared with all other sequential single lung transplants performed. Solid line represents actuarial survival for the CF recipients receiving bilateral sequential single lung transplants (BSLTx); broken line represents all other BSLTx performed at our centre $(n=100)$. No significant difference in survival was found between the cohorts (log rank testing; Mantel-Cox, $\mathrm{p}=0.29$ ). 
Table 1 Comparison of published actuarial survival figures for lung transplantation in cystic fibrosis recipients from selected case series

\begin{tabular}{|c|c|c|c|c|c|c|c|}
\hline & Newcastle & $\begin{array}{l}\text { Newcastle } \\
\text { (excl. } \\
\text { BCC) }\end{array}$ & Egan $^{17}$ & Burton $^{5}$ & Quattrucci $^{9}$ & $\begin{array}{l}\text { Hadjiliadis }^{16} \\
\text { (sens) }\end{array}$ & $\begin{array}{l}\text { Hadjiliadis }{ }^{16} \\
\text { (PanR) }\end{array}$ \\
\hline $1 \mathrm{y}$ & 82 & 85 (134) & 81 & 89 & 79 & 96 & 88 \\
\hline $3 y$ & 70 & 72 (97) & NR & 80 & 70 & 91 & 63 \\
\hline $5 y$ & 62 & 62 (63) & 59 & 80 & 58 & 85 & 58 \\
\hline $8 y$ & 56 & 56 (33) & NR & 70 & NR & NR & NR \\
\hline $10 y$ & 51 & 53 (19) & 38 & NR & NR & NR & NR \\
\hline$n=$ & 176 & 157 & 123 & 36 & 55 & 58 & 45 \\
\hline
\end{tabular}

Newcastle survival data at 1 year $(1 \mathrm{y})$. Newcastle (excluding BCC) represents our transplantation results for CF excluding those with pretransplantation Burkholderia cepacia complex (BCC) infection (includes bilateral sequential single lung transplants and other operative interventions). The actual number of patients surviving at each time point is noted in italics. Data reported elsewhere include Egan and colleagues, ${ }^{17}$ Burton and colleagues ${ }^{5}$ and Quattrucci and colleagues. ${ }^{9}$ Hadjiliadis et al represents survival outcomes in cystic fibrosis recipients with sensitive organisms (sens) and panresistant organisms (PanR). ${ }^{16}$ n, recipient numbers, NR, not recorded.

oncology services. Minor skin neoplasia rates caused by basal cell carcinoma or superficial squamous cell carcinomas were not actively recorded.

\section{RESULTS}

Lung transplantation commenced at this institution in 1987 with the first transplantation procedure for CF in 1989. The total number of lung transplantation procedures performed at the time of writing was 575 performed for all indications, with 176 lung transplantations performed in patients with CF $(30 \%$ of the total lung transplant population). Of these, 167 were adults (age $>17$ years) and nine were paediatric cases (age $<17$ years; range 12.5-17). There were 91 female patients and 85 male patients transplanted. Median age at transplantation was 26.2 years (range 12.5-49.5).

The operative interventions were 168 bilateral (sequential) lung transplantations from cadaveric donors (BSLTx), four heart-lung transplantation procedures, one patient received living related lobar donor transplantation and in two cases single lung transplantation. One patient underwent BSLTx and combined liver transplantation. Additionally, one CF recipient who had previously undergone BSLTx had "re-do" single lung transplantation performed for acute respiratory failure requiring intubation and mechanical ventilation This patient died after the re-do transplant within 30 days, and as a result we no longer retransplant patients who are invasively ventilated. In contrast our experience with re-do transplantation, for other indications transplanted when stable, has a mean survival of more than 2 years.

In those recipients receiving single lung transplantation, one patient had previously had a pneumonectomy and the other had single lung transplantation and contralateral pneumonectomy because of thoracic wall deformity. ${ }^{11}$ Nineteen patients had pretransplantation infection with BCC and were grouped together in view of the association with poorer outcomes following transplantation. The outcomes in this group will be discussed in detail elsewhere in terms of microbiology and genomovar related outcomes (in submission).

\section{Pretransplantation morbidity}

Significant pretransplantation morbidity was noted within this group; 71 patients $(40 \%)$ had pretransplantation diabetes mellitus, the majority of whom were treated with insulin therapy. A further 35 patients (20\% of cohort) developed diabetes following transplantation, leaving 61 (35\%) at followup with normal serum glucose levels and normal HbA1c levels, with $5 \%$ of patients awaiting annual assessment. The majority of patients developing post-transplantation diabetes were established on insulin therapy (31/35). In those who developed diabetes following transplantation, 31 patients were maintained on tacrolimus at the time of our assessment and four remained on ciclosporin.

As previously reported, pleural disease was also common. ${ }^{12}$ We noted 21 patients (12\%) with a previous pneumothorax. The pretransplantation management by referring teams included intercostal chest drain in 11 patients, medical pleurodesis in four patients and thoracic surgical pleurodesis in two patients. Survival outcomes were not worsened in those with previous pneumothorax (Mantel-Cox log rank testing $\mathrm{p}=0.78) .{ }^{12}$ An additional patient had undergone upper lobectomy prior to transplant listing. Previous non-invasive ventilation had been used in 22 patients (12\% of the cohort). Prior non-invasive ventilation was not associated with a poorer prognosis compared with the rest of the CF cohort (Mantel-Cox log rank testing, $p=0.89$ ). Percutaneous enterogastrostomy was present in 23 recipients (13\%) pretransplantation and overnight nasogastric feeding in 10 recipients (6\%). Median body mass index was $18.6 \mathrm{~kg} / \mathrm{m}^{2}$ pretransplantation (range 12-26) and this increased at 1 year to a median of $20.3 \mathrm{~kg} / \mathrm{m}^{2}$.

\section{Survival and causes of death}

The survival figures for the whole cohort were $82 \%$ survival at 1 year, $70 \%$ at 3 years, $62 \%$ at 5 years and $51 \%$ at 10 years. Our survival figures, excluding those patients with pretransplant BCC infection, were $84 \%$ survival at 1 year, $70 \%$ survival at 3 years, $60 \%$ survival at 5 years and $53 \%$ at 10 years. We compared our Kaplan-Meier survival rates for the CF cohort with that of bilateral (sequential) lung transplantation for all other transplantation indications at our centre (fig 1, table 1). There was no significant difference noted between survival in $\mathrm{CF}$ and non-CF transplantation cohorts (log rank testing; Mantel-Cox, $\mathrm{p}=0.29$ ).

There are currently 107 CF lung transplant recipients alive (61\% of cohort). Causes of death were not identifiable in all cases. This reflects our wide referral area and shared care with referring centres of post-transplant patients. Three patients died at home or at the referring centre and did not have satisfactory data to confirm the recorded cause of death was correct. We were able to identify 35 cases where obliterative bronchiolitis or BOS was the recorded cause of death, accounting for $51 \%$ of all observed deaths (table 2). 
Table 2 Causes of death in cystic fibrosis recipients transplanted at the Freeman Hospital Transplant Programme

\begin{tabular}{ll}
\hline Cause of death & $\begin{array}{l}\text { No of patients } \\
(\%)\end{array}$ \\
\hline Obliterative bronchiolitis & $35(51)$ \\
Infection & $18(26)$ \\
$B$ cepacia complex & $7(10)$ \\
Cytomegalovirus & $2(3)$ \\
C difficile & $2(3)$ \\
Aspergillus spp & $1(1.5)$ \\
$P$ aeruginosa & $1(1.5)$ \\
Unknown & $5(7)$ \\
Respiratory failure & $2(3)$ \\
Malignancy & $2(3)$ \\
ARDS & $2(3)$ \\
Graft dysfunction & $2(3)$ \\
Gastrointestinal bleed & $1(1.5)$ \\
PTLD & $1(1.5)$ \\
Cardiac arrest & $1(1.5)$ \\
Bronchial dehiscence & $1(1.5)$ \\
Neurological complications & $1(1.5)$ \\
Unknown & $3(4.5)$ \\
Total No of deaths & $69(100)$ \\
\hline Causes of death in recipients were recorded from case notes. Data \\
are expressed as percentage of all deaths observed in this cohort. \\
Thirty-five cases of obliterative bronchiolitis were identified and this \\
accounted for 51\% of all deaths in the cohort. The percentages have \\
been rounded up or down to the first decimal place. \\
ARDS, acute respiratory distress syndrome; PTLD, post-transplant \\
lymphoproliferative disorder.
\end{tabular}

Death caused by sepsis was noted in 18 cases (of these BCC infection was the cause of death in seven cases, Clostridium difficile colitis in two cases, cytomegalovirus infection in two cases, panresistant Pseudomonas aeruginosa in one case and Aspergillus infection in one case). In an additional three cases, a sepsis syndrome was identified as the cause of death although no specific pathogen was isolated. Our current sepsis death rate was therefore $26 \%$ of all recorded CF transplantation recipient deaths. Other causes of death were rare $(n=1$ unless otherwise stated). These included respiratory failure $(n=2)$, primary graft dysfunction $(n=2)$, acute respiratory distress syndrome $(n=2)$, post-transplant lymphoproliferative disease $(n=1)$, other malignancies (lung $(\mathrm{n}=1)$ and liver $(=1)$ ), neurological complications, cardiac arrest, bronchial dehiscence and portal hypertension associated gastrointestinal bleeding.

\section{Pulmonary function and obliterative bronchiolitis}

Pulmonary function $\left(\mathrm{FEV}_{1} \%\right.$ predicted) improved from a pretransplantation median of 0.81 (21\% predicted) to 2.951 at 1 year following transplantation (78\% predicted). The median $\mathrm{FEV}_{1}$ at 5 years post-transplantation was $2.75 \mathrm{I}$ and $2.2 \mathrm{l}$ at 10 years post-transplantation $(p<0.01$ at each time point compared with pretransplantation values, paired $t$ test). Obliterative bronchiolitis, as defined clinically by BOS status, was BOS 0 (freedom from BOS) ${ }^{9}$ in $85 \%$ at 1 year, $74 \%$ at 5 years and $38 \%$ at 10 years (fig 2).

\section{Renal disease}

Pretransplantation EDTA-GFR results demonstrated a median GFR of $125 \mathrm{ml} / \mathrm{min}$ (range 67-200). Following transplantation, serum creatinine worsened with a mean pretransplant creatinine of $73 \mathrm{mg} / \mathrm{dl}$ (range 33-140) rising to $121.5 \mathrm{mg} / \mathrm{dl}$ (range $66-235)$ at 1 year $(p<0.001)$ (fig 3$)$. Estimated GFR (eGFR) data

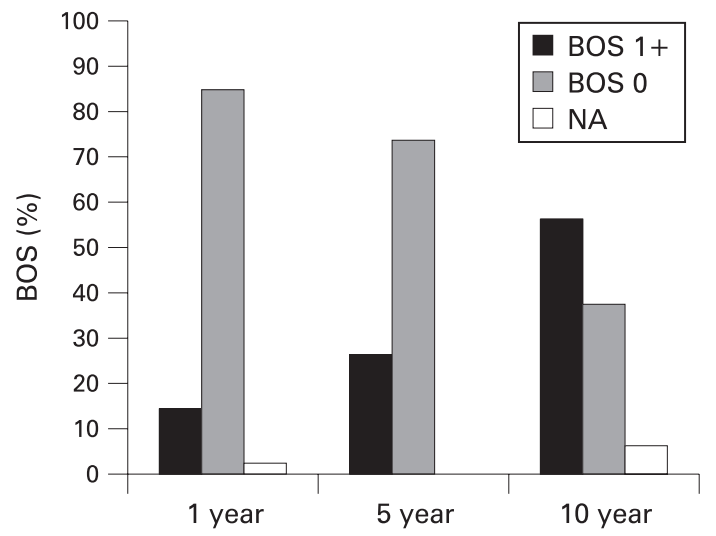

Figure 2 Rates of bronchiolitis obliterans syndrome (BOS) in cystic fibrosis recipients. Freedom from BOS at 1 year $(n=134), 5$ years $(n=63)$ and 10 years $(n=19)$ following transplantation in CF recipients with BOS grade 1 or higher, CF recipients who had "freedom from BOS" and in patients where data were not available (NA).

are not available at the time of writing, reflecting our institution's recent adoption of this measurement. Three patients required haemodialysis $(1.1 \%$ of the total CF transplant cohort-one patient remains alive). Two patients have undergone secondary renal transplantation $(1.1 \%$ of total CF transplant cohort-one patient remains alive).

\section{Surveillance associated comorbidities}

The majority of patients underwent our standard biopsy protocols resulting in more than 800 transbronchial biopsy procedures performed. No patient died as a consequence of a biopsy procedure. No cases were noted as requiring invasive mechanical ventilation or blood transfusion following a transbronchial biopsy. Reflecting the recently instituted prospective electronic database of biopsy results, we identified 438 biopsies taken within the first year. Acute vascular rejection (International Society for Heart Lung Transplantation (ISHLT) grade A2 or greater) was noted in 77 of 187 biopsies taken within the first month (0-30 days). Thus biopsy data gave an observed first month acute vascular rejection rate of $41 \%$. We identified 32/251 cases of acute rejection from days 30-366 posttransplantation as part of our routine surveillance programme.

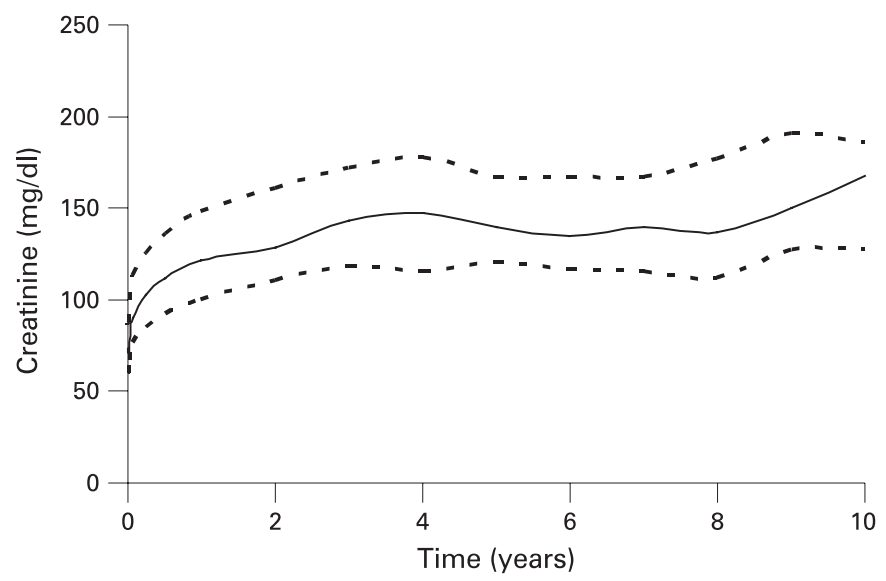

Figure 3 Change in serum creatinine $(\mathrm{mg} / \mathrm{dl})$ following transplantation. Solid line indicates the median creatinine level observed and the broken lines represent the 25th and 75th centile creatinine levels. 


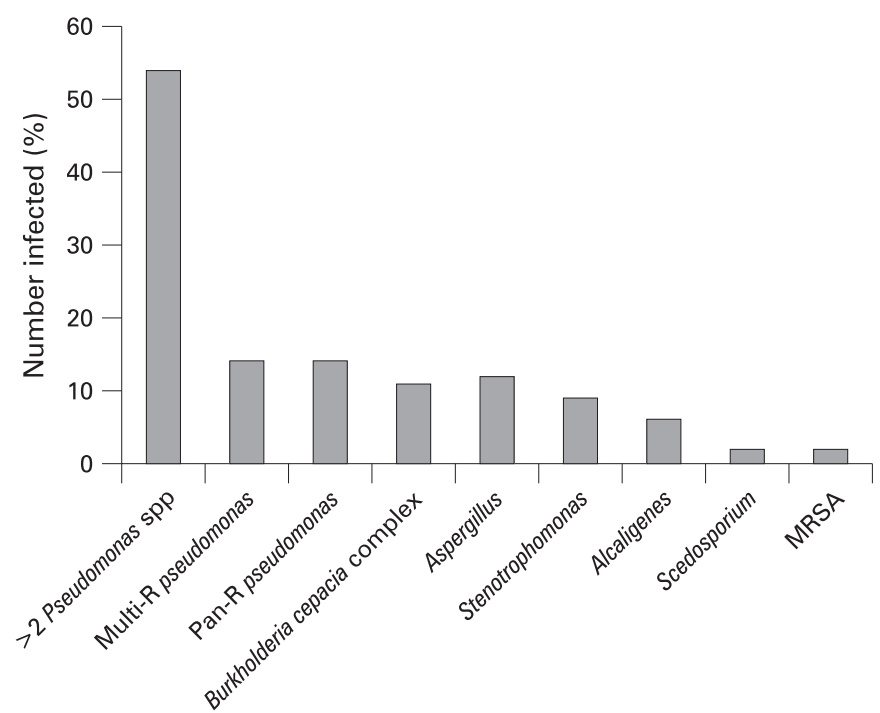

Figure 4 Microbial infections prior to transplantation. Percentage of the cohort infected with each pathogen is noted. Coinfection was common, with many patients having polymicrobial infections. The first histogram represents patients infected with more than one Pseudomonas species or strain. Multiresistance (Multi-R) and panresistant (pan-R) was as defined previously. ${ }^{10}$ MRSA, methicillin resistant $S$ aureus.

This rejection rate equates to $12 \%$ of biopsies undertaken between 1 month and 1 year post-transplantation.

\section{Malignancy}

One patient suffered donor acquired small cell lung carcinoma, as previously reported. ${ }^{13}$ Post-transplant lymphoproliferative disease was noted in five patients (2.8\% of cohort).

\section{Microbiology}

Peritransplantation microbiological cultures, including those prior to transplantation and within the first week following transplantation, were achieved in all patients. A large range of pathogens were seen in the cohort and polymicrobial infections in individual recipients were common (fig 4). Infection with more than one Pseudomonas strain was assumed in the presence of mucoid and non-mucoid phenotypes and/or widely differing antibiotic sensitivities. Pulsed field gel electrophoresis studies that assess different bacterial lineages were not routinely conducted so our assumption may be incorrect and such bacteria could include some clonal strains with marked morphotypic/phenotypic variation. Noting this caveat, we found $54 \%$ of patients appeared to have two or more distinct Pseudomonas strains. A further $14 \%$ of patients had panresistant Pseudomonas aeruginosa infections and similarly $14 \%$ of patients had multiresistant Pseudomonas aeruginosa infections. BCC infections were noted in 15\%, Aspergillus infection in $12 \%$, Stenotrophomonas spp infection in $9 \%$, Alcaligenes spp infections in $6 \%$, Scedosporium spp infections in $2 \%$ and methicillin resistant $S$ aureus infection (MRSA) in 2\%. Atypical mycobacteria were not isolated in our cohort at the time of transplantation although infection with such organisms does not reflect a specific exclusion criterion for transplantation at this centre.

\section{Airway complications}

In 176 recipients with 346 bronchial anastomoses, we noted four recipients with major anastomotic complications (overall major bronchial anastomotic complication rate $2 \%$ ). One recipient suffered bronchial dehiscence. Three recipients had anastomotic strictures that required dilatation and stenting.

\section{DISCUSSION}

Lung transplantation for CF accounts for approximately onethird of all sequential single lung transplants performed at this centre, a distribution similar to that reported by the International Society for Heart Lung Transplantation (ISHLT) registry. ${ }^{1}$ Our series of cystic fibrosis lung transplants is one of the largest reported to date and our survival rates are similar to the best of the previously reported series (table 1). ${ }^{10}{ }^{14-16}$ The survival rates for CF recipients reported by Egan et al were similar to those at our centre, most procedures were bilateral sequential single lung transplantation, and were $81 \%$ at 1 year, $59 \%$ at 5 years and $38 \%$ at 10 years. ${ }^{17}$ Our approach has been predominantly with bilateral sequential single lung transplantation and this compares favourably with that of heart-lung transplantation for CF. ${ }^{18}$

The predominant surgical technique used was bilateral sequential single lung transplantation with cardiopulmonary bypass. Routine use of bypass facilitated a standard technique across a relatively large surgical and anaesthetic team. Both main bronchi were stapled after recipient pneumonectomy, facilitated by the single lumen endotracheal tube which allowed irrigation and removal of contaminated secretions from the (retained) distal airway. We believe this technique reduces intraoperative contamination. Finally, reperfusion of both lungs simultaneously allows close control of pressure and avoids circulatory overload of one lung.

Bronchial anastomotic complications were uncommon, with an overall major bronchial anastomotic complication rate of $2 \%$ in comparison with prior reports in CF recipients of up to $7 \%$ and $14 \% .{ }^{19}{ }^{20}$ More recently, rates of anastomotic complications in a cohort, including CF and other lung transplantation indications, were $15 \%{ }^{21}$ The reasons for our low anastomotic complication rates remain unclear. We did not, however, routinely perform bronchial artery revascularisation (possible protective benefits $)^{22}{ }^{23}$ nor did we routinely perform telescoping of the bronchial anastomosis (possible promoter of anastomotic complications). ${ }^{21}$ We do not however have data on other factors recently implicated in the genesis of anastomotic complications, such as recipient length and donor ventilation times. ${ }^{21}$ Despite our low rates of anastomotic complications, one episode was felt to have directly contributed to the recipient's death.

We noted a high rate of pathogens with potential to complicate the early postoperative period, including panresistant Pseudomonas aeruginosa, BCC and Aspergillus species. We compared our CF cohort with that of all other bilateral sequential lung transplants performed at our centre (including non-CF bronchiectasis, sarcoidosis, emphysema) and found no significant differences in survival rates (log rank testing $\mathrm{p}=0.29$ ) Confounding variables between CF recipients and non-CF recipients may however include differing recipient ages and comorbidities in these two cohorts.

Most of the early deaths (deaths within the first 30 days) following lung transplantation for CF were felt to be caused by sepsis. We noted that $26 \%$ of our CF recipient deaths were a result of sepsis, which is also comparable with or less than that reported from other centres. ${ }^{6}{ }^{14}$ There were significantly higher mortality rates in those who had preoperative BCC infection where seven of 19 patients died from sepsis (36\%). The observed higher risk of death due to sepsis in those with pretransplantation BCC infection confirms that of prior reports demonstrating 
excessive mortality in BCC infected patients. ${ }^{14}{ }^{24}$ Our full experience of transplantation with BCC infection and genomovar specific outcomes will be discussed elsewhere (in press).

No other organisms were significantly associated with poor transplantation outcomes. In particular, infection with multior panresistant Pseudomonas aeruginosa had no survival disadvantage, confirming prior findings ${ }^{10} 2526$ but in contrast with a more recent publication. ${ }^{16}$ None of our recipients had pretransplantation infection with non-tuberculous mycobacteria. This does not reflect a specific policy of declining to transplant individuals with such infections although we do not transplant those infected with $M$ abscessus because of concerns over posttransplantation morbidity. ${ }^{27}$

We did not prospectively assess the clonal nature of bacterial strains with pulsed field gel electrophoresis and assumed that strains with markedly different antibiograms and morphotypes were unrelated clonally. This phenotypic approach may overestimate the number of distinct species of organisms or strains isolated noted in fig 4.

Pretransplantation comorbidity was common, with significant rates of CF related diabetes, CF related liver disease, prior pneumothorax or chest surgery and weight loss. We do not view CF related liver disease as a contraindication per se although we regularly seek expert assessment for hepatobiliary and liver transplant colleagues. Despite this multidisciplinary approach, one patient died of hepatobiliary complications related to variceal bleeding following transplantation. Low body mass index was noted in selected patients with the lowest recorded pretransplantation body mass index of $12 \mathrm{~kg} / \mathrm{m}^{2}$. In general, we prefer to avoid transplanting patients with a body mass index less than $16 \mathrm{~kg} / \mathrm{m}^{2}$ but may elect to do so if the potential recipient has no other relative contraindications and has made maximal efforts with assisted nutrition. Interestingly, there was no clear association between poor post-transplant survival and prior pneumothorax or prior non-invasive ventilation.

We noted a good functional outcome following transplantation with an increase in $\mathrm{FEV}_{1}$ at 1 year following transplantation to almost 31 (78\% predicted). Similar to prior reports, we used "freedom from BOS", as defined by patients who failed to demonstrate a fall in $\mathrm{FEV}_{1}$ to the criterion used for BOS 1 or higher. ${ }^{15}$ The majority of patients were free from BOS 1 or higher at 1 year $(\sim 85 \%)$ and $73.6 \%$ at 5 years. In those recipients surviving 10 years, $37.5 \%$ of patients had freedom from BOS 1. Similar "freedom from BOS" rates in CF recipients have been reported at the Rome transplant programme (95\% at 1 year, $82.5 \%$ at 2 years, $70 \%$ at 3 years and $65 \%$ at 5 years). ${ }^{9}$ Earlier reports have demonstrated freedom from BOS as $84 \%$ at 1 year and $51 \%$ at 3 years. ${ }^{15}$ One possible criticism using "freedom from BOS" and not the newly categorised BOS 0-p is that our study cannot report those at risk of BOS. We were unable to report BOS 0 -p because of the large numbers of patients being transplanted prior to the introduction of the new BOS 0-p category where $\mathrm{FEF}_{25-75}$ data were unavailable.

Transplantation in our CF cohort was associated with significant post-transplantation comorbidities, in particular renal dysfunction. ${ }^{2}$ In a smaller series previously reported, three patients (11\% of the total CF recipients) required dialysis following lung transplantation. In the series of 55 Italian CF recipients reported by Quattrucci and colleagues, ${ }^{9}$ the authors noted that the most common medical complication after transplantation, observed in 27 patients, was chronic renal failure. Our rates of renal dysfunction are therefore lower than those previously reported. This is noteworthy as we now have an aggressive policy in non-rejecters (those with biopsies of grade A1 or less) to reduce target ciclosporin or tacrolimus levels by the end of the first year. Such a strategy may however be ineffective as the majority of nephron loss has been postulated to occur in the first year following transplantation. ${ }^{28}$ While aggressive aminoglycoside antibiotic therapy may stabilise patients with CF on a transplant waiting list, we did not specifically assess if those patients with lower transplant assessment EDTA-GFR recordings had received more courses of these potentially nephrotoxic antibiotics. Irrespective of this, the level of post-transplantation renal dysfunction in this cohort has remained low.

Other important major post-transplantation complications encountered in our cohort have included four recipients who developed fulminant pseudo-membranous colitis where the mortality rate was $50 \%$ despite urgent colectomy. ${ }^{29}$ One patient has required a post-transplantation left pneumonectomy related to pulmonary vascular anastomosis compromise associated with mediastinal lymphadenopathy.

Post-transplantation bronchoscopy and surveillance associated morbidity was extremely low and had a high detection rate of asymptomatic acute vascular rejection similar to that recently reported. ${ }^{30}$ Many of these rejection episodes were asymptomatic and not associated with an obvious fall in lung function. The surveillance protocols used at our centre have also contributed to our understanding of post-transplantation graft dysfunction offering hope in extending the longevity of both the graft and recipient. ${ }^{31-33}$

The retrospective nature of our study has, by definition, inherent weaknesses. Many of the deaths were not confirmed by autopsy findings leading to potential incorrect categorisation of the cause of death (eg, BOS may be associated with secondary bacterial infection) and this makes the cause of death difficult to differentiate. Autopsy should be considered where possible if the cause of decline is not clear. Also of note is the lack of prospective data on metabolic bone disease, a common comorbid condition in CF. Although we are unaware of any known osteoporotic fractures, a systematic bone density assessment programme following transplantation was not adopted until recently. Other important areas to be considered for future studies would include employment status, assessments of psychiatric morbidity and quality of life measurements.

Bilateral sequential lung transplantation is a well established treatment for patients with end stage CF associated lung disease. The perioperative mortality is low and recipients with CF have a significant early survival and functional benefit after lung transplantation. Long term results are good, although both transplantation associated comorbidities and increasing rates of graft dysfunction occur with time.

Funding: ADS was supported by a HEFCE Senior Lectuereship.

Competing interests: None.

\section{REFERENCES}

1. Trulock EP, Edwards LB, Taylor DO, et al. Registry of the International Society for Heart and Lung Transplantation: twenty-third official adult lung and heart-lung transplantation report-2006. J Heart Lung Transplant 2006;25:880-92.

2. Bloom RD, Doyle AM. Kidney disease after heart and lung transplantation. Am J Transplant 2006;6:671-9.

3. Aurora $\mathbf{P}$, Whitehead $\mathbf{B}$, Wade $A$, et al. Lung transplantation and life extension in children with cystic fibrosis. Lancet 1999;354:1591-3.

4. Mariencheck WI Jr, Palmer SM, Tapson VF, et al. Survival following lung transplantation of cystic fibrosis patients colonized with Burkholderia cepacia. Am J Respir Crit Care Med 2000;161:720.

5. Burton CM, Milman N, Carlsen J, et al. The Copenhagen National Lung Transplant Group: survival after single lung, double lung, and heart-lung transplantation. J Heart Lung Transplant 2005;24:1834-43. 
6. de Perrot M, Chaparro C, McRae K, et al. Twenty-year experience of lung transplantation at a single center: Influence of recipient diagnosis on long-term survival. J Thorac Cardiovasc Surg 2004;127:1493-501.

7. Aaron SD, Ferris W, Henry DA, et al. Multiple combination bactericidal antibiotic testing for patients with cystic fibrosis infected with Burkholderia cepacia. Am J Respir Crit Care Med 2000;161:1206-12.

8. Lang BJ, Aaron SD, Ferris W, et al. Multiple combination bactericidal antibiotic testing for patients with cystic fibrosis infected with multiresistant strains of Pseudomonas aeruginosa. Am J Respir Crit Care Med 2000;162:2241-5.

9. Quattrucci S, Rolla M, Cimino G, et al. Lung transplantation for cystic fibrosis: 6-year follow-up. J Cyst Fibros 2005;4:107-14.

10. Aris RM, Gilligan PH, Neuringer IP, et al. The effects of panresistant bacteria in cystic fibrosis patients on lung transplant outcome. Am J Respir Crit Care Med 1997;155:1699-704.

11. Forty J, Hasan A, Gould FK, et al. Single lung transplantation with simultaneous contralateral pneumonectomy for cystic fibrosis. J Heart Lung Transplant 1994; 13:727-30.

12. Curtis HJ, Bourke SJ, Dark JH, et al. Lung transplantation outcome in cystic fibrosis patients with previous pneumothorax. J Heart Lung Transplant 2005;24:865-9.

13. De Soyza AG, Dark JH, Parums DV, et al. Donor-acquired small cell lung cancer following pulmonary transplantation. Chest 2001;120:1030-1.

14. Chaparro C, Maurer J, Gutierrez C, et al. Infection with Burkholderia cepacia in cystic fibrosis; outcome following lung transplantation. Am J Respir Crit Care Med 2001;163:43-8.

15. Egan TM, Detterbeck FC, Mill MR, et al. Lung transplantation for cystic fibrosis: effective and durable therapy in a high-risk group. Ann Thorac Surg 1998;66:337-46.

16. Hadjiliadis D, Steele MP, Chaparro C, et al. Survival of lung transplant patients with cystic fibrosis harboring panresistant bacteria other than Burkholderia cepacia, compared with patients harboring sensitive bacteria. J Heart Lung Transplant 2007;26:834-8.

17. Egan TM, Detterbeck FC, Mill MR, et al. Long term results of lung transplantation for cystic fibrosis. Eur J Cardiothorac Surg 2002;22:602-9.

18. Ganesh JS, Rogers CA, Bonser RS, et al. Outcome of heart-lung and bilateral sequential lung transplantation for cystic fibrosis: a UK national study. Eur Respir J 2005;25:964-9.

19. Mendeloff EN. Lung transplantation for cystic fibrosis. Semin Thorac Cardiovasc Surg 1998;10:202-12.
20. Venuta F, Quattrucci S, Rendina EA, et al. Improved results with lung transplantation for cystic fibrosis: a 6-year experience. Interact Cardiovasc Thorac Surg 2004;3:21-4.

21. De Wauwer C, Van Raemdonck D, Verleden GM, et al. Risk factors for airway complications within the first year after lung transplantation. Eur J Cardiothorac Surg 2007;31:703-10

22. Norgaard MA, Olsen PS, Svendsen UG, et al. Revascularization of the bronchial arteries in lung transplantation: an overview. Ann Thorac Surg 1996;62:1215-21.

23. Hyytinen TA, Heikkila LJ, Verkkala KA, et al. Bronchial artery revascularization improves tracheal anastomotic healing after lung transplantation. Scand Cardiovasc J 2000;34:213-18.

24. Aris RM, Routh JC, LiPuma JJ, et al. Lung transplantation for cystic fibrosis patients with Burkholderia cepacia complex. Survival linked to genomovar type. Am J Respir Crit Care Med 2001;164:2102-6.

25. Kanj SS, Tapson V, Davis RD, et al. Infections in patients with cystic fibrosis following lung transplantation. Chest 1997:112:924-30.

26. Dobbin C, Maley M, Harkness J, et al. The impact of pan-resistant bacterial pathogens on survival after lung transplantation in cystic fibrosis: results from a single large referral centre. J Hosp Infect 2004;56:277-82.

27. Chernenko SM, Humar A, Hutcheon M, et al. Mycobacterium abscessus infections in lung transplant recipients: the international experience. J Heart Lung Transplant 2006;25:1447-55

28. Canales M, Youssef $P$, Spong $R$, et al. Predictors of chronic kidney disease in long-term survivors of lung and heart-lung transplantation. Am J Transplant 2006:6:2157-63.

29. Yates B, Murphy DM, Fisher AJ, et al. Pseudomembranous colitis in four patients with cystic fibrosis following lung transplantation. Thorax 2007;62:554-6.

30. Benden C, Harpur-Sinclair 0, Ranasinghe AS, et al. Surveillance bronchoscopy in children during the first year after lung transplantation: Is it worth it? Thorax 2007;62:57-61.

31. De Soyza A, Fisher AJ, Small T, et al. Inhaled corticosteroids and the treatment of lymphocytic bronchiolitis following lung transplantation. Am J Respir Crit Care Med 2001;164:1209-12.

32. Ward C, De Soyza A, Fisher AJ, et al. A descriptive study of small airway reticular basement membrane thickening in clinically stable lung transplant recipients. J Heart Lung Transplant 2005:24:533-7.

33. Ward C, De Soyza A, Fisher AJ, et al. Reticular basement membrane thickening in airways of lung transplant recipients is not affected by inhaled corticosteroids. Clin Exp Allergy 2004;34:1905-9.

\section{Lung alert}

\section{IL6 and dendritic cells in allergic asthma}

Interleukin (IL)6 production by antigen-presenting dendritic cells (DCs) is integral to the differentiation of $\mathrm{T}$ helper cells into $\mathrm{T}$ helper type Th1, Th2 and Th17 subsets. However, molecular mechanisms that regulate IL6 production in DCs are yet to be elucidated. Stimulation of bone marrow-derived dendritic cells with the allergen house dust mite (HDM) - which causes asthma in humans - or the mucosal adjuvant cholera toxin promoted cell surface expression of cKit and its ligand, stem cell factor (SCF), resulting in sustained signalling downstream of c-Kit, prompting IL6 secretion. DCs from c-Kit mutant mice secreted smaller amounts of IL6 following HDM or cholera toxin stimulation, resulting in an inability to induce a robust Th2 or Th17 response and reduced allergic airway inflammation. In addition, expression of Jagged-2, a Notch ligand which has been associated with Th2 differentiation, is reduced in DCs lacking functional c-Kit, implying that c-Kit upregulates expression of Jagged-2 in addition to IL6. Cell signalling downstream of c-Kit is likely to involve phosphorylation of Akt via activation of phosphatidylinositol-3 kinase (PI3k), as DCs expressing a catalytically inactive form of PI3K secrete less IL6 on cholera toxin stimulation.

It is concluded that cell surface expression of c-Kit by DCs in conjunction with SCF causes extended activation of the PI3K-Akt pathway, promoting a higher IL6 expression profile in DCs which, in turn, promotes T cell differentiation towards Th2 and Th17 lineage. This may have implications for the understanding and treatment of allergic airway inflammation in asthma as downregulation of c-Kit may reduce such inflammation. Inhibition of c-Kit may also promote the efficacy of vaccines in cancer therapy by augmenting a Th1 response.

- Krishnamoorthy N, Oriss TB, Paglia M, et al. Activation of c-Kit in dendritic cells regulates Thelper cell differentiation and allergic asthma. Nat Med 2008;14:565-73.

\section{E Fletcher}

Correspondence to: C E Fletcher, MRes Biomedical Research, Imperial College, London SW7 2AZ, UK; claire.fletcher07@ imperial.ac.uk 